\title{
EL ASPECTO Y SU PROYECCIÓN EN LA SINTAXIS DE LAS CLÁUSULAS SINTÉTICAS SUBCATEGORIZADAS
}

\author{
Ángel Jiménez Fernández
}

\begin{abstract}
In this article we intend to show that the distribution of predicates in Small Clauses is determined by semantics, not by syntax. Our framework is Chomsky's (1995) Mnimalist Program. Each predicate is given an aspectual feature in the Lexicon which must be compatible with the semantic nature of the matrix verb in an appropriate context. We claim that this aspectual information is projected in the syntactic representation of the structures under study by the insertion of the syntactic category of ASP(ect), which must contain the same aspectual feature as the Small Clause predicate in order for the derivation to converge.
\end{abstract}

\section{Introducción}

El objetivo del presente trabajo es dar cuenta de los requisitos de selección sintácticosemántica entre un verbo matriz y una C(láusula) S(intética) subcategorizada (Small Clause) que nos ayude a discernir el tipo de predicado que puede aparecer en ésta. El marco teórico en el que nos basamos es el Programa Minimalista de Chomsky (1995).

Dentro de nuestra hipótesis se encuentra la propuesta de que el Aspecto influye de manera decisiva en la derivación óptima de una cláusula, entendiendo ASP como categoría sintáctica independiente. La información aspectual aparece en la entrada léxica de algunas piezas junto a su red temática. Conjuntamente conforman la información léxica que se proyecta en la sintaxis (Demonte 1991; De Miguel 1992), hipótesis a la que nos adherimos. Sin embargo, reelaboraremos esta idea para explicar ciertos contrastes gramaticales que muestran las CSs argumentales.

La propuesta concreta que queremos formular es que la habilitación de los tipos de predicados en las CSs subcategorizadas depende crucialmente del rasgo aspectual que requieren los predicados matrices. Esta hipótesis nos llevará a incluir en la estructura de la CS la categoría funcional ASP, especificada con los rasgos [ \pm perfectivo], que legitima la proyección que toma por complemento. 


\section{Aclaraciones Preliminares sobre el Aspecto}

En nuestra propuesta trataremos el aspecto de forma globalizadora (Comrie 1976; Rojo 1988; De Miguel 1992), frente a la división que se hace en la literatura entre aspecto y Aktionsart (Bache 1982). Efectivamente podríamos hablar de un aspecto morfológico, subjetivo en la medida en que cada hablante ve el modo de ser del proceso verbal a su manera, y un aspecto léxico, objetivo pues es intrínseco a la entrada léxica de cada predicado.

A efectos de nuestra propuesta parece no ser necesaria esta distinción, aunque a veces recurramos a ella. Principalmente nuestro estudio pretende formalizar el tipo de relación existente entre el aspecto léxico de una pieza y su habilitación como predicado de una CS subcategorizada. El contenido aspectual de una entrada léxica se debe proyectar de alguna manera en la sintaxis mediante la inserción de un S(intagma) Asp(ectual) en la derivación de una cláusula.

Mucha confusión ha creado el término aspecto en la literatura, cuestión que trata De Miguel (1992); sin embargo, tampoco la división entre predicados perfectivos y predicados imperfectivos está exenta de incoherencias, que querremos esclarecer. La dicotomía [ \pm perfectivo] se suele tomar en relación al hecho de si el evento denotado por el predicado está delimitado o no (Tenny 1987), o si el evento se ve como permanente o transitorio (Demonte 1991). No obstante, no parece haber acuerdo entre los autores sobre si un predicado perfectivo es permanente, no delimitado e inacadado (Bosque 1990; Hernanz 1988), o si dicho predicado perfectivo es temporal, delimitado y acabado (Demonte 1991; De Miguel 1992).

Nosotros, al igual que Bosque (1990), nos vamos a guiar por la etimología del término "perfectivo". Así un predicado perfectivo será aquel que exprese un evento sujeto a cambio, que se puede completar, mientras que un predicado imperfectivo implica un evento constante, ya completado. En otras palabras, "perfectivo" significará en este trabajo "perfeccionable", delimitable, temporal, susceptible a cambio.

El rasgo [ \pm perfectivo] será en nuestro marco teórico un valor semántico seleccionador de una categoría compatible con dicho rasgo. Mantenemos que en el PM las categorías que una estructura desarrolla en su derivación deben cumplir una serie de requisitos de selección. Una representación se forma mediante la regla de ensamble (merging), la cual se aplica por razones de selección. De ahí que los valores aspectuales conlleven una compatibilización semántica.

Siguiendo líneas trazadas por Tenny (1987), mantenemos que la información aspectual hace de intermediaria entre el Lexicón y la sintaxis. En el Lexicón los predicados -tanto verbos como adjetivos, sustantivos y preposiciones- vienen marcados inherentemente con los rasgos [ \pm perfectivo]. En la selección de un predicado para entrar en la derivación de una cláusula influyen estos rasgos, de forma que a la hora de expandirse una CS en el SAsp 
el rasgo de perfectividad ha de ser compatible con el mismo valor que contiene el núcleo ASP.

A veces el ASP será neutral, aceptando como complemento una CS cuyo predicado sea perfectivo o imperfectivo; otras veces el ASP estará determinado por el verbo matriz, de forma que éste exija un predicado perfectivo o no perfectivo en la CS.

(1) a. I consider him dead/honest.

b. I expect him dead/*honest.

El hecho diferencial radica en que honest es un predicado especificado con el rasgo [perfectivo], compatible con el rasgo neutral de ASP que permite consider en (1a), pero no con el rasgo [+ perfectivo] que selecciona expect. Por el contrario, dead posee el valor [+ perfectivo], pudiéndose legitimar cuando el ASP de la CS es neutral como en (1a), y también cuando ASP se manifiesta como [+ perfectivo] como en (1b).

La diferencia que estamos trazando entre predicados perfectivos e imperfectivos ha tenido un correlato en otros términos. Así, Chung y McCloskey (1987) y Raposo y Uriagereka (1990 y 1995) distinguen entre Individual-Level small clauses y Stage-Level small clauses. Las primeras reciben una interpretación donde la propiedad asignada por el predicado de la CS se ve como permanente, mientras que las segundas poseen un predicado que denota una propiedad transitoria.

Raposo y Uriagereka (1990) demuestran que los verbos de percepción toman sólo CSs Stage-Level, mientras que los verbos de opinión seleccionan únicamente CSs IndividualLevel. Sin embargo, esta división no puede explicar por qué ambos ejemplos en (2) son gramaticales:

(2) a. I consider the sea/the frog green.

b. I saw the sea/the frog green.

Nótese que green denota una propiedad constante y según Raposo y Uriagereka (1995), debería aparecer sólo con una CS Individual-Level. Éste es el caso de (2a), pero también este predicado puede aparecer con un verbo de percepción física; entonces cómo dar cuenta de la gramaticalidad de $(2 \mathrm{~b}) ?^{1}$

Parece que la solución no es pragmática y mucho menos simplemente sintáctica (Stowell 1983). La selección entre predicado matriz y predicado subordinado está mediatizada por la compatibilidad entre el rasgo [ \pm perfectivo] de la categoría ASP y el

\footnotetext{
${ }^{1}$ A partir de los hechos rese ados en (2), Raposo y Uriagereka (1995) siguen por unos derroteros de índole pragmática que no vamos a considerar aquí.
} 
mismo rasgo del predicado de la CS. En otras palabras, la hipótesis que estamos formulando supone que es la selección semántica entre ASP y predicado subordinado la que dicta la convergencia de la derivación. Ciertamente Hale y Keyser (c.p.) confirman que parece sentirse algún tipo de rasgo aspectual en las CSs argumentales asociado a distintas clases de predicados.

La propuesta de que el aspecto condiciona la habilitación de ciertas construcciones no es nueva. Demonte (1991), Hernanz (1988) y Bosque (1990) demuestran que la predicación secundaria está limitada por el rasgo aspectual [ \pm perfectivo] del predicado de la CS adjuntiva. Sin embargo, Hernanz sugiere que las CS argumentales no están constre idas por el aspecto.

Bosque (1990:204-205) menciona que verbos como querer en español sí seleccionan aspectualmente una clase determinada de predicado en la CS que subcategoriza. De ahí que surja la diferencia entre las oraciones en (3):

(3) a. Quiero el coche reparado para el martes.

b. Lo quiero más limpio.

c. *María quiere falso el resultado.

No obstante, predicados matrices como considerar o juzgar contrastan claramente con querer ya que, aunque Bosque no lo examina, aceptan un estado permanente o transitorio dentro de la CS.

\section{Compatibilidad de los Rasgos [ \pm Perfectivo] en los Predicados de las CSs.}

Hemos asumido que la información léxica de cada pieza engloba tanto su red temática como una serie de rasgos entre los que destaca la valencia aspectual (Demonte 1991; Hernanz 1988; De Miguel 1992). El predicado de una CS no va a ser una excepción y la interacción de la red temática y la perfectividad es, a nuestro entender, la encargada de legitimar o discriminar la coaparición de un predicado matriz y ciertos predicados en la CS correspondiente.

En esta sección queremos demostrar que lo que los predicados matrices requieren de su CS subcategorizada es que el predicado de ésta sea compatible aspectualmente con el rasgo de la misma índole que aquellos poseen, basándonos en la dicotomía $\left[ \pm\right.$ perfectivo]. ${ }^{2}$

\footnotetext{
${ }^{2}$ Bosque (1990) lanza un postulado parecido. Este autor demuestra que las CSs adjuntivas deben contener en aspecto [+ perfectivo] compatible con el predicado matriz. Nuestra propuesta pretende explicar las restricciones aspectuales de las CSs argumentales. Con respecto a estas últimas, Bosque defiende que también deben estar especificadas como [+ perfectivo], lo cual no da cuenta del predicado [- perfectivo] en She considers him intelligent.
} 
Para la aplicación de esta clasificación aspectual y sus consecuencias empíricas vamos a partir de las oraciones en (4) y (5), que Stowell (1983) usa para mostrar que el predicado matriz selecciona la categoría sintáctica del predicado de la CS. También hemos de tener en cuenta las oraciones (6) y (7) de Kitagawa (1985), ya que constutuyen los primeros indicios de que la selección entre predicado matriz y subordinado no es sintáctica sino semántica.

(4) a. I consider John very stupid.

b. I expect that sailor off my ship by midnight.

(5) a. *I consider John off the ship.

b. *I expect that sailor very stupid.

(6) a. *The doctor considers that patient dead tomorrow.

b. Unfortunately, our pilot considers that island off the route.

(7) a. *I expect that island off the route.

b. I expect that man dead by tomorrow.

Las oraciones (4) y (5) llevan a Stowell (1983) a concluir que existe selección sintáctica entre predicado matriz y predicado subordinado. Esta conclusión es revocada por Kitagawa (1985), el cual mencionaba las oraciones (6) y (7) como elementos de juicio para sostener que la selección es semántica. De lo contrario, no se podría explicar que en ciertos contextos consider subcategorice una CS cuyo predicado sea un SP -(6b)- o expect requiera una CS con un SA como predicado -(7b)-.

Kitagawa (1985) proponía que la diferencia semántica radica en que consider selecciona un estado, mientras que expect selecciona un cambio de estado. Pollard y Sag (1990) examinan estos hechos y deducen que entre predicado matriz y predicado subordinado media no sólo selección semántica sino también sintáctica. Al insertar la cópula be, los ejemplos agramaticales serían totalmente aceptables:

(8) a. I expect that island to be a good vacation spot.

b. *I expect that island a good vacation spot.

Si expect no tiene acceso vía subcategorización a la categoría sintáctica del predicado de la CS, cómo explicar el contraste (8a-b)?

La idea que nosotros queremos defender comparte con Kitagawa (1985) la asunción de que la diferencia entre estado y cambio de estado juega un papel fundamental a la hora de dar licencia gramatical a una CS argumental. Sin embargo, se aparta de este autor en muchas de las consecuencias que éste deriva de su propuesta. Principalmente, el hecho de 
que consider acepte un estado expresado por el predicado de la CS no elimina la posibilidad de aparecer con un cambio de estado, tal como se aprecia en (19):

(9) I consider the tank completely full.

Tampoco las consecuencias empíricas que nosotros deducimos de las diferencias semánticas que muestran las CSs coinciden, como se verá, con las hipótesis de Kitagawa. Por una parte, la división entre estado y cambio de estado no es, a nuestro juicio, tan tajante. Podríamos distinguir estadios intermedios como estados susceptibles al cambio o estados de cambio. El predicado full en (9) parece expresar esta característica. De otra parte, esta división de estados tiene un origen aspectual, en tanto se basa en la capacidad de cambio de una propiedad, cuestión en la que no entra Kitagawa (1985). Estas divergencias aspectuales tendrán su correlato en la estructura sintáctica que conlleva la derivación de la CS argumental.

Siguiendo a Tenny (1987) y Demonte (1991), podemos distinguir estados indivisibles en subestados y estados que son divisibles en un estadio inicial, medio o interno y final. Los predicados perfectivos serán estos estados divisibles en estadios, los cuales implican o pueden potencialmente implicar un cambio. Aquí radica la diferencia entre cambio de estado y estado de cambio o, en otras palabras, estado alcanzado y estado alcanzable. La cuestión es que tanto uno como otro son predicados marcados con el rasgo aspectual [+ perfectivo]. Ejemplos de este grupo son full, broken, empty, in good form, out of his mind, amused, pleased, etc.

Contrariamente, aquellos estados que presentan una unidad atómica -es decir, los no divisibles en estadios- constituyen los estados simples. No son eventos que se puedan completar y por tanto, estarían aspectualmente especificados con el valor [- perfectivo], como sucede con intelligent, modest, honest, false, true, a good teacher, etc.

Esta clasificación está basada en la que ofrece Hernanz (1988:17) sobre los adjetivos predicativos en espa ol. Esta autora propone una tercera clase que estaría especificada con ambos rasgos [ \pm perfectivo], ya que aspectualmente estos adjetivos funcionan de manera ambivalente. Este tipo engloba predicados como fat, thin, clean, dirty, crazy, attractive, elegant, etc. Uno de los criterios que usa Hernanz para clasificar los predicados es su capacidad de coaparición con las cópulas serlestar, las cuales serían indicadores de perfectividad, tal como se puede ver al parangonar las oraciones en (11):

(11) a. María es gorda. [- perf]

b. María está gorda. [+ perf]

Esta dicotomía copulativa no se da en inglés, al menos en los mismos términos, por lo que no nos parece un criterio acertado. Además, un SP como fuera de la ruta estaría marcado siempre como [- perfectivo], pues sólo puede acompa ar a la cópula estar. Esto sería indicativo de que designa un estado alcanzado o potencialmente alcanzable: 
(12) Esa escala está fuera de la ruta.

No obstante, este SP puede habilitarse como predicado de una CS argumental seleccionada por verbos como considerar, que pueden requerir un estado simple y entonces el predicado SP tendría la valencia [- perfectivo]:

(13) Considero esa escala fuera de la ruta.

Parece que el marcado aspectual intrínseco de ciertas piezas léxicas puede variar dependiendo de ciertas circunstancias. Principalmente, un rasgo aspectual puede modificarse según si el predicado matriz requiera un estado o un cambio de estado y si en el contexto en cuestión se favorece una determinada interpretación. Lo que queremos afirmar es que la habilitación de un predicado viene condicionada no sólo por el marcado aspectual inherente, sino también por un marcado aspectual dictado por el contexto. Tal como nos confirma Uriagereka (c.p.), cualquier categoría sintáctica puede aparecer como predicado de la CS si el contexto así lo permite. De aquí se deriva que la correlación entre perfectividad y la dicotomía serlestar no sea buen diagnóstico para legitimar o discriminar ciertas construcciones. ${ }^{3}$ Así, en inglés no podemos dar cuenta de la diferencia del rasgo aspectual del predicado subordinado en cada uno de los siguientes ejemplos:

(6) b. Unfortunately, our pilot considers that island off the route. [- perf]

(14) I expect that island off the route by tomorrow. [+ perf]

Nosotros hemos distinguido entre estados imperfectivos y estados perfectivos, a la vez que asumimos con Hernanz (1988) y Demonte (1991) la idea de que un SD expresa siempre inherentemente un estado acabado, una propiedad permanente y por consiguiente se especificaría en el Lexicón con el rasgo [- perfectivo] ${ }^{4}$. El SA y el SP son ambivalentes en tanto que pueden expresar una propiedad transitoria o permanente.

Por otro lado, entendemos que determinados contextos hacen que un predicado cambie su valencia aspectual. Así, por ejemplo, happy estaría marcado léxicamente como [-

${ }^{3}$ Un predicado como feliz puede aparecer tanto con ser como con estar según el contexto:

(i) María es feliz de la vida.

(ii) María está feliz de la vida.

(iii) Considero a María feliz de la vida (ahora).

El predicado de la CS en la oración (iii) también puede interpretarse como estado simple o cambio de estado. Según Kitagawa (1985), consider sólo admite un estado en la CS. Cómo podemos explicar, entonces, el hecho de que en (iii) el predicado subordinado se pueda interpretar como un cambio de estado que se ha producido en María? Nótese que la adición del adverbio favorece esta interpretación perfectiva.

${ }^{4}$ Existen SDs que pueden comportar un cambio de estado. Lo que sucede es que en ciertos contextos la valencia intrínseca [- perfectivo] de un SD puede ser modificada. Esto es lo que ocurre en She made me a good husband. El SD a good husband implica normalmente un estado simple, pero en una CS seleccionada por un verbo causativo cormo make el mismo predicado subordinado pasa a denotar un estado alcanzado. 
perfectivo], capacitándose como predicado de una CS seleccionada por un verbo con la misma valencia aspectual, tal como consider:

(15) a. I consider him happy.

No obstante, happy puede aparecer con el rasgo [+ perfectivo], implicando que es un estado alcanzado, completado, que conlleva un estadio anterior no tan feliz y, por tanto, esta pieza puede habilitarse con un predicado especificado como [+ perfectivo] o [perfectivo].

(15) b. I will make you happy.

c. I consider him happy now although he has gone through so much pain.

De otra parte, existen predicados matrices que son aspectualmente neutros. Consider puede coaparecer con un predicado subordinado especificado como [+ perfectivo] o [perfectivo]. Otros predicados matrices sólo aceptan una CS cuyo predicado esté marcado en un único sentido.

Esto no quiere decir que la habilitación de las CSs argumentales no esté restringida aspectualmente, tal como defiende Hernanz (1988), sino que a diferencia de la predicación secundaria, los predicados de las CSs subcategorizadas pueden ser [+ perfectivo] o [perfectivo] dependiendo del contexto que determine el predicado matriz que selecciona a la CS.

Con esta descripción podemos ya explicar contrastes como los de (16):

(16) a. I consider him intelligent.

b. *I'll make him intelligent.

c. She makes me happy.

d. She considers me happy.

En estas oraciones aparecen los verbos matrices consider y make, cuya diferencia aspectual radica en que make sólo acepta una CS que incluya un predicado con el rasgo [+ perfectivo] -de ahí la agramaticalidad de (16b)-, mientras que consider puede seleccionar una CS con un predicado [+ perfectivo] o [- perfectivo]. Por esta razón, junto a las oraciones (16a) y (16d), también podemos se alar la gramaticalidad de (17):

(17) a. I consider the agreement broken. [+ perf]

b. I consider Mary very worried about her job. [+ perf]

Mientras que en (16a) y (16d) el predicado que aparece en la CS es [- perfectivo], en $(17 \mathrm{a}-\mathrm{b})$ los predicados broken y worried mostrarían la valencia [+ perfectivo]. En 
consecuencia, consider es un verbo marcado doblemente como [ \pm perfectivo] en cuanto al predicado subordinado que parece seleccionar.

Con respecto a las oraciones en (2), repetidas a continuación,

(2) a. I consider the sea/the frog green.

b. I saw the sea/the frog green.

hay que destacar que un predicado como green puede estar especificado como [+ perfectivo] o [- perfectivo]. Demonte (1991:122) arguye que los adjetivos que denotan. colores son intrínsecamente [- perfectivo] y, contra Hernanz (1988), pueden predicarse secundariamente.

(18) José compró el coche verde y lo vendió marrón.

Sin embargo, tanto el uso de marrón en (18) como de green en (2b) suponen la evidencia de que estos adjetivos pueden tener la valencia [+ perfectivo], ya que en ambos casos se requiere un predicado designador de un cambio de estado. Lo que queremos afirmar es que los colores se encuentran marcados ambivalentemente en lo que al aspecto se refiere, eligiéndose un rasgo u otro según el predicado matriz en cuestión.

Esta ambivalencia aspectual explica la aparición de green en (2a) donde se puede entender que la rana o el mar es verde o se han puesto verde, pues consider admite predicados que implican un estado permanente o un cambio de estado. Frente a esto, $(2 b)$ sólo puede interpretarse correctamente si green tiene el rasgo [+ perfectivo], significando que es una característica transitoria, perfectamente compatible con el cambio que selecciona el predicado matriz see en este caso. Recuérdese la importancia del contexto en la compatibilización de los rasgos aspectuales, como nos corrobora Uriagereka (c.p.).

En lo que concierne al contraste en (3),

(3) a. Quiero el coche reparado para el martes.

b. Lo quiero más limpio.

c. *María quiere falso el resultado.

parece que la perfectividad también es la causa de la agramaticalidad de (3c). El predicado querer requiere una CS cuyo predicado denote un cambio de estado, sea éste actual o potencial. Por esta razón, en (3a-b) los predicados reparado y limpio, marcados como [+ perfectivo], pueden coaparecer con el predicado matriz querer. No obstante, falso sólo puede tener la valencia aspectual [- perfectivo] y en consecuencia, se produce un conflicto de rasgos entre predicado matriz y predicado subordinado que hace que (3c) sea inaceptable. 
Los ejemplos en (4-5) y (6-7) también muestran que es la selección semántica la que filtra el tipo de predicado que manifiesta la CS argumental. Comenzando con el predicado matriz consider, en (4a) lo encontramos con un predicado [- perfectivo] en John very stupid, mientras que en (5a) y (6a) los predicados de la CS, off the ship y dead suponen una propiedad transitoria que los marca como [+ perfectivo], desechándose su presencia en un contexto donde consider requiere un estado simple.

Sin embargo, si contextualizamos la oración (5a) en una situación que implique un cambio de estado con respecto a otro estado anterior, la agramaticalidad desaparece (Hornstein y Lightfoot 1987):

(19) As soon as he sets foot on the gangplank, I'll consider John off the ship.

En este contexto el juicio de considerar se ve en el futuro, suponiendo que se considera un estado que ha cambiado en relación a otro estado. En esta situación consider puede seleccionar una CS cuyo predicado sea [+ perfectivo].

En (6a) hay adicionalmente un conflicto temporal entre el momento en que se considera y el intervalo denotado por el adverbial tomorrow en que se muere el paciente. De nuevo, en un contexto apropiado esta oración sería gramatical.

(20) Doctors say she won't live one more day, so I will consider her dead by tomorrow.

Con respecto a los ejemplos (4b), (5b), (7) y (19), hemos de se alar que expect es un predicado matriz que selecciona una CS con un predicado [+ perfectivo]. Este predicado subordinado debe designar una propiedad transitoria. De ahí el contraste entre (4b) y (5b). Esto no significa que la CS subcategorizada por expect deba contener un SP y no un SA, tal como arguye Stowell (1983), ya que en (7) observamos que mientras el SA dead puede aparecer en la CS, el SP off the route no es compatible con el cambio de estado que requiere expect, a menos que se contextualice como en (13), donde sí se presupone este cambio.

En conclusión, parece que existen predicados matrices marcados como [+ perfectivo], [perfectivo] o [ \pm perfectivo], al tiempo que los predicados de la CS deben estar especificados con el mismo rasgo. La restricción general es que predicado matriz y predicado subordinado deben ser aspectualmente compatibles en un contexto apropiado.

Ahora podemos entender que quizás el hecho de que verbos como consider admitan cualquier predicado en su CS haya llevado a ciertos lingüistas a postular que las CSs argumentales no experimentan ninguna restricción de orden aspectual. Lo que sucede realmente es que consider es aspectualmente polifacético y sus construcciones son gramaticales tanto si el predicado subordinado es [+ perfectivo] como si es [- perfectivo], todo supeditado al contexto interpretativo. 
Queda aún una cuestión pendiente: por qué cuando se inserta to be no hay diferencia entre consider y expect? En otras palabras, cómo explicar el contraste en (8)?

(8) a. I expect that island to be a good vacation spot.

b. *I expect that island a good vacation spot.

De la taxonomía aspectual que hemos propuesto y su relación con las categorías sintácticas, resulta claro que (8b) es inaceptable porque un SD predicativo suele expresar una propiedad constante, marcándose en el Lexicón con el rasgo [- perfectivo], y expect selecciona un cambio de estado que no puede proporcionar el SD a good vacation. La lectura que hacen Pollard y Sag (1990:4) del contraste en (8) es que el predicado de la CS es accesible a las propiedades de subcategorización de expect, mientras que el predicado de la cláusula de infinitivo no está subcategorizado por el verbo matriz.

La lectura que nosotros hacemos es que en (8a) la selección aspectual queda interrumpida o, mejor, neutralizada por la presencia de to be. Si parangonamos las oraciones espa olas en (21),

(21) a. Considero que esa isla es un buen sitio de vacaciones.

b. Espero que esa isla sea un buen sitio de vacaciones.

podemos apreciar la diferencia entre el modo indicativo (real) y el modo subjuntivo (irreal o futurible). (21b) expresa un cierto estado sujeto a variación que presupone un estado anterior, tal vez dudoso, que se consigue mediante el modo subjuntivo de la cópula. Es decir, la valencia aspectual inherente del SD un buen sitio de vacaciones puede verse afectada por el cambio potencial que manifiesta la cópula.

En inglés es sabido que no existe apenas la distinción entre indicativo y subjuntivo. Por esta razón, parece que (8a) tiene las mismas implicaciones que (22):

(22) I consider that island to be a good vacation spot.

No obstante, una mirada más atenta revela la diferencia. En (22) el aspecto de la cláusula de infinitivo sigue siendo [- perfectivo], mientras que en (8a) está marcado como [+ perfectivo]. El aspecto perfectivo exigido por expect a su complemento es asignado mediante la misma marca de la cópula, bajo la asunción de que el verbo es el portador de la perfectividad.

\section{Proyección del ASP(ecto) en la Sintaxis de las CSs Subcategorizadas.}

Hemos se alado el papel tan importante que los rasgos aspectuales [ \pm perfectivo] adquiere en la legitimación de las CSs argumentales. Ciertos predicados matrices requieren que el predicado de su CS connote bien un estado simple, bien un cambio de estado. En esta 
sección queremos formalizar esta selección semántico-aspectual en la derivación sintáctica de estas construcciones dentro del PM.

A modo de introducción, afirmaremos que tanto el aspecto morfológico como el aspecto léxico se proyectan en la sintaxis y asumiremos la existencia de un núcleo funcional ASP que filtra los rasgos aspectuales de los predicados de las CSs argumentales. ${ }^{5}$ Esta observación quedará corroborada por la distribución de los adverbios aspectuales, aquellos que modifican la temporalidad interna del predicado en cuestión y que se adjuntan al hipotético SAsp (Demonte 1991; Ojea López 1997).

Partiendo de una estructura del tipo de la de Stowell (1983), sostenemos que la secuencia [SD SX] constituye el complemento del SAsp, lo cual explica los requisitos de perfectividad en términos configuracionales. Bosque (1990) propone una estructura parecida con respecto a la predicación secundaria, aunque para éste el SAsp debe ser siempre perfectivo. Como veremos, éste no es el caso en las CSs argumentales.

Para demostrar la necesidad de postular un núcleo ASP en la estructura de una CS argumental que justifique las diferencias semántico-aspectuales encargadas de la distribución de los predicados en este tipo de cláusulas, necesitamos mostrar alguna evidencia de que las CSs incluyen este nudo aspectual. Un buen diagnóstico para probar la existencia de una categoría sin representación léxica consiste en la aceptabilidad de adverbiales que se generan adjuntados a dicha categoría, de forma que este adverbial cumpla sus requisitos de alcance (Ojea López 1997:174).

En la literatura se asume que existen adverbiales que modifican a ciertas categorías funcionales. Así, con respecto al ASP, podemos distinguir una serie de adverbios aspectuales que hacen más explícita la temporalidad interna que describe un evento. Demonte (1991:147) hace una diferencia entre adverbios temporales, aspectuales y los del modo de la acción. Las dos últimas clases son las que nos interesan.

Dentro de los adverbios aspectuales Demonte incluye recién, ya, de una vez, hace un momento, todavía o continuamente; entre los adverbios de la Aktionsart estarían a menudo, con frecuencia, dos veces, muchas veces o siempre.

A su vez, Bosque (1990:193-194) a ade a los adverbios aspectuales piezas como completamente, enteramente, y del todo, los cuales modifican a predicados que denotan

\footnotetext{
${ }^{5}$ Sobre ASP como categoría funcional que desarrolla el aspecto léxico, ver Bosque (1990) Hernanz (1988). De Miguel (1992) y Demonte (1991) postulan un concepto unitario de aspecto por el que tanto el morfológico como el léxico están incluidos en el núcleo ASP.

Nosotros hemos asumido este tratamiento unitario del aspecto. Obviamente, como se demostrará, nuestra propuesta aunque basada parcialmente en la que formulan estos lingüistas, se diferenciará tanto en mecanismos técnicos como en consecuencias teórico-empíricas.
} 
procesos que se pueden completar. Por esta razón, sólo aparecen con predicados que manifiestan el rasgo [+ perfectivo]:

(23) a. Completamente vacío, enteramente seco, limpio del todo.

b. *Completamente bueno, *enteramente alto, *corpulento del todo.

Lo mismo ocurre con adverbios aspectuales de la talla de una vez y ya, los cuales se alan el estado inmediatamente posterior al obtenido por el proceso denotado por el predicado (De Miguel 1992; Bosque 1990). Estos adverbios sólo se adjuntan a predicados especificados con la valencia [+ perfectivo]:

(24) a. Una vez vacía, ya fijo, etc.

b. *Una vez inteligente, *ya sensacional, etc.

Resulta natural extender esta restricción aspectual a todos los adverbios de aspecto, ya que en ningún caso un estado simple puede ir acompa ado por éstos. De lo contrario, ese estado simple quedaría dividido en varios subestados, lo cual no es posible. Sólo los estados alcanzados o alcanzables -los que incluyen el rasgo [+ perfectivo]- pueden coaparecer con adverbios aspectuales.

Por otro lado, Ojea López (1997:177) plantea un concepto unitario de los adverbios aspectuales en inglés, no marcando una distinción entre adverbios del modo de acción y adverbios aspectuales. Para lo que queremos demostrar aquí, no necesitamos ser tan minuciosos, en tanto que unos y otros se adjuntarían al mismo nudo, SAsp. Los adverbios aspectuales que Ojea López analiza son: always, ever, never, already, still, yet, frequently, often, normally, sometimes, generally, usually, scarcely, rarely, etc.

Lo que nos interesa de los adverbios aspectuales es su posibilidad de manifestación en una CS argumental. Si podemos probar la presencia de al menos ciertos adverbios aspectuales en la CS, ello constituirá un argumento para postuliar una categoría ASP a la que se deben adjuntar dichos adverbios. Los siguientes ejemplos avalan nuestras conjeturas:

(25) a. I find [him often trustworthy].

b. I find [him always in good humour].

c. ?I consider [him usually clumsy].

d. I consider [it already empty].

El hecho de que los adverbios already, usually, often, always, etc. puedan habilitarse en las CSs argumentales justifica la presencia de la categoría ASP en las mismas. Nótese también que estos adverbios aspectuales aparecen en CSs cuyo predicado es [+ perfectivo], de lo contrario la construcción sería inaceptable: 
(26) a. *I consider [him usually tall].

b. *I find [him often a good man].

c. *I find [it already perfect].

Los predicados subordinados en (26) incluyen el rasgo aspectual [- perfectivo], lo que los incapacita para ser modificados por un adverbio aspectual debido a su incompatibilidad léxico-semántica.

Si observamos ejemplos como (25c), marcado como dudoso, podemos apreciar que el predicado clumsy tiene intrínsecamente la valencia [- perfectivo]. Por tanto, (25c) debería ser agramatical. Sin embargo, en un contexto adecuado esta oración podría considerarse aceptable. Esto es debido al fenómeno antes aludido de que el contexto puede variar el valor aspectual de [- perfectivo] a [+ perfectivo]. Así, clumsy en (25c) debe entenderse como un estado transitorio y por tanto alcanzado. Esta propiedad queda plasmada en espa ol en el contraste que sigue:

(27) a. *Considero que él es normalmente torpe.

b. Consider que él está normalmente torpe.

Aunque el paralelismo entre aspecto y la dicotomía serlestar no es muy fiable, al comparar las oraciones en cuestión concluimos que (25c) sería aceptable con la interpretación [+ perfectiva] de (27b), pero agramatical si se descifra con la lectura no perfectiva de (27a).

De lo expuesto en torno a la presencia de la categoría ASP y los adverbios aspectuales en una CS argumental se deduce que la CS debe estar dominada por un SAsp. En términos más rigurosos, una vez que se han seleccionado los elementos léxicos que componen la CS junto con sus rasgos gramaticales y semánticos, se forma una estructura del tipo de la de Stowell (1983), que se inserta en la derivación como complemento del SAsp. Obtenemos el siguiente resultado:

$$
\begin{array}{r}
\text { (28) [SAsp Espec [ASP' ASP [sX SUJ [x' X ] ] ] }] \\
{[ \pm \text { perf }]} \\
{[ \pm \text { perf }]}
\end{array}
$$

Es esta configuración la consecuencia del primer paso de la derivación en el sistema de cómputo, sea cual sea la categoría sintáctica del predicado de la CS y su proyección, de ahí el término genérico $\mathrm{SX}$.

Una de las propiedades de selección consiste en la compatibilidad de rasgos entre un núcleo y su complemento (Chomsky 1995), que se manifiesta mediante el ensamble de categorías. Esta particularidad se plasma en la derivación (28) en la relación entre el núcleo ASP y su compiemento SX. Míás estrictamente, el rasgo aspeciual de ÁSP déve ser 
compatible con el mismo rasgo en $\mathrm{X}$, de lo contrario el ensamble no se produciría y la representación no llegaría a formarse (Demonte (c.p.)).

Otra propiedad que se ala la derivación (28) es que se pueden establecer varias clases de relaciones aspectuales entre ASP y SX. Si el predicado posee el rasgo [- perfectivo], el ASP también incluirá el mismo rasgo. Esto explica la aceptabilidad de (4a):

\section{(4) a. I consider [ ASP John very stupid]}

$$
\text { [- perf] [- perf] }
$$

Cuando el predicado está especificado como [+ perfectivo], el ASP también se marcará con la misma valencia, de forma que no se produzca un conflicto de rasgos entre núcleo y complemento. Así damos cuenta del contraste que sigue:

(7) a. I expect [ ASP that man dead by tomorrow]

$$
[+ \text { perf }] \quad[+ \text { perf }]
$$

(5) b. *I expect [ ASP that sailor very stupid]

$$
\text { [+ perf] [- perf] }
$$

La derivación de (5b) se estrella debido al desajuste de los rasgos aspectuales de ASP y del SA very stupid, que hace que no se ensamblen las categorías ASP y el SA predicado.

Por último, un predicado puede tomar ambos valores, en cuyo caso ASP debe ser neutral. En estas ocasiones ASP puede contener los rasgos [+ perfectivo] o [- perfectivo] indistintamente. Por esta razón, las oraciones en (15) son estructuras convergentes:

(15) a. I consider [ ASP him happy]

$$
[- \text { perf }] \quad[- \text { perf }]
$$

c. I consider [ ASP him happy], although he has gone through so much pain.

$$
[+ \text { perf }] \quad[+ \text { perf }]
$$

La cuestión es qué condiciona la categoría ASP para que contenga un rasgo u otro, a la vez que seleccione un predicado especificado en un determinado sentido. La respuesta está en la naturaleza aspectual del predicado matriz.

Existen verbos que exigen un estado simple, un cambio de estado o, a veces, ambos. Si tomamos, por ejemplo, el verbo expect podemos apreciar que este predicado determina el ASP perfectivo de la CS, la cual sólo podrá admitir un predicado marcado con el rasgo [+ perfectivo]. Consider, sin embargo, puede condicionar el ASP de la CS en cualquiera de los dos términos de la oposición dependiendo del contexto. 
En (4a) consider selecciona un ASP no perfectivo, mientras que el predicado subordinado en (15) permite que esta selección aspectual esté constre ida por el contexto. Razonablemente, podemos considerar un estado simple, al tiempo que podemos también considerar un estado alcanzado. Se puede ser feliz o estar feliz en un momento determinado. Ésta es la diferencia que se da entre (15a) y (15c).

\section{Conclusiones}

En este trabajo hemos demostrado que las restricciones aspectuales constri en el tipo semántico de predicado que puede aparecer en una CS argumental. No se trata de una selección sintáctica, sino semántica entre el verbo matriz y el predicado de la CS en términos aspectuales. Esta selección está mediatizada por una categoría funcional ASP que debe contener un rasgo compatible con el del predicado que la complementa. El rasgo de ASP viene determinado por el verbo matriz y condicionado por el contexto.

Las restricciones aspectuales se proyectan en la sintaxis, más concretamente en la representación de una estructura, haciendo que mediante el mecanismo de ensamble el verbo matriz selecciona a ASP y éste a un predicado aspectualmente afín, de forma que si no se cumplen los requisitos de ensamble la representación no llega a formarse y la estructura es agramatical.

\section{Referencias}

Bache, C., "Aspect and Aktionsart: Towards a Semantic Distinction", Journal of Linguistics 18, (1982) 57-72.

Bosque, I., "Sobre el Aspecto en los Adjetivos y en los Participios", en I. Bosque (ed.) Tiempo y Aspecto en Espa ol. Madrid: Cátedra. (1990) 177-214.

Chomsky, N., The Minimalist Program. Cambridge, Mass.: MIT. (1995).

Chung, S. y J. McCloskey, "Government, Barriers, and Small Clauses in Modern Irish", Linguistic Inquiry 18 (1987) 173-237.

Comrie , B., Aspect: An Introduction to the Study of Verbal Aspect and Related Problem. Cambridge: C.U.P. (1976).

Demonte, V., Detrás de la Palabra: Estudios de Gramática del Espa ol. Madrid: Alianza Editorial. (1991).

Hernanz, M.L., "En Torno a la Sintaxis y la Semántica de los Complementos Predicativos en Espa ol", Estudi Generali 8 (1988) 7-29. 
Kitagawa, Y., "Small but Clausal", Chicago Linguistic Society 21 (1985) 210-220.

Miguel, E. de, El Aspecto en la Sintaxis del Espa ol: Perfectividad e Impersonalidad. Madrid: Ediciones UAM. (1992).

Ojea López, A., (1997) "La Distribución de los Adverbios en Inglés", Actas del XVIII Congreso de AEDEAN, Universidad de Alcalá de Henares. (1997) 171-180.

Pollard, C. y I.A. Sag, "An Information-Based Syntax and Semantics", Vol. 2: Topics in Control and Binding. CSLI Lecture Note Series. Stanford: CSLI. (1990).

Raposo, E. y J. Uriagereka, "Long-Distance Case Assignment", Linguistic Inquiry 21, (1990) 505-537.

Raposo, E. y J. Uriagereka, "Two Types of Small Clauses (Toward a Syntax of Theme/Rheme Relations", en: A. Cardinaletti y M.T. Guasti (eds.), Syntax and Semantics: Small Clauses. 179-206. (1995)

Rojo, G., "Temporalidad y Aspecto en el Verbo Espa ol", LEA X (1988) 195-216.

Stowell, T., "Subjects across Categories", The Linguistic Review 2 (1983) 285-312.

Tenny, C., Grammaticalizing Aspect and Affectedness. Tesis Doctoral inédita, MIT. (1987). 
